\title{
Individuelle Schreiblernberatung. Einzelfalldarstellung am Beispiel der Abteilung für Deutschlehrerausbildung der Universität Trakya ${ }^{1}$
}

\author{
Çağlayan Karaoğlu Bircan (iD, Edirne - Handan Köksal (iD, Edirne \\ https://doi.org/10.37583/diyalog. 1030764
}

\begin{abstract}
Deutsch)
In der letzten Zeitwende haben sich viele Forscher den neuesten zeitgenössischen Sprachlernmethoden und Techniken für einen effektiveren Sprachunterricht gewidmet. Diese Veränderungstendenzen in Lehr- und Lernpädagogik erfordern in ihrer Umsetzung die individuelle Führung des Lerners. In diesem Zusammenhang entwickeln sich Konzepte der Beratungslehre. Unter diesem Aspekt wurde unter einer 12 Wochen langen, vielseitigen Forscher/ Berater-Beobachtung im Studienjahr 2018-2019 eine Aktionsforschung in der Deutschlehrerausbildungsabteilung der Universität Trakya in Edirne durchgeführt. Beabsichtigt wurde dabei, eine institutionell eingerahmte, professionell konzipierte Sprachlernberatung mit Fokus Schreiben zu realisieren. Fernerhin wird in dieser Forschung der Problemfrage, ob DaF-Lerner durch eine individuelle Schreiblernberatung ihren Schreibprozess fördern können, nachgegangen. Die Schreiblernberatungen werden nach einem von der Forscherin eigenständig gestalteten Model durchgeführt. Die Datensammlungen verlaufen zweiteilig quantitativ und qualitativ. Die Resultate der Forschung zeigen, dass von der Sprachlernberatung profitiert wird, diesbezüglich Blockaden und Defizite beim Deutsch Schreiben behoben werden. In diesem weiterführenden Beitrag ${ }^{2}$ werden anhand eines Fallballspieles der Forschungsprozess und die Ergebnisse repräsentativ dargelegt.
\end{abstract}

Schlüsselwörter: Schreiben, individuelle Führung, Lernberatung, Sprachlernberatung, Schreiblernberatung.

\section{Abstract (English) \\ Individual Writing Counselling. Case Study Using the Example of The University of Trakya, German Teacher Training}

In the last era, many researchers have devoted themselves to the latest contemporary language learning methods and techniques for more effective language teaching. These change tendencies in teaching/ learning pedagogy require the individual guidance of the learner in their implementation. In this context, concepts of counselling are developing. With this in mind, an action research is being carried out in the German teacher training department of the University of Trakya in Edirne in the academic year 2018-2019 as part of a dissertation work under a 12-week long, versatile researcher/ consultant observation. The

Einsendedatum: 04.06.2021

Freigabe zur Veröffentlichung: 01.12.2021

${ }^{1}$ Extrakt aus der Dissertation „Sprachlernberatung in der Deutschlehrerausbildung Schreiben im Fokus“.

2 Die genannte Dissertation wurde mit dem Titel „Sprachlernberatung in der Deutschlehrerausbildung Schreiben im Fokus“ an der Deutschlehrerausbildung (DLAA) der Fremdsprachenabteilung des Instituts für Sozialwissenschaften an der Trakya Universität unter der Leitung von Prof. Dr. Handan Köksal erstellt. 
intention is to carry out an institutionally framed, professionally designed language learning consultation (LLC) with a focus on writing. This selection is based on the fact that the writing process of foreign language learners is often a problem area in language learning. As a result, the research investigates the problem of whether German training learners can promote their writing process through individual writing counselling, which are carried out according to a model that the researcher designed independently. The data collections for the implementation are two-part, quantitative and qualitative. The results of the research show that has been benefited from the LLC and that blockages and deficits in German writing are eliminated. In this further contribution, the research process and the results are presented in a representative manner using a case study.

Keywords: Writing, Individual Guidance, Language Learning Counselling, Writing Counselling. 


\section{EXTENDED ABSTRACT}

Recently, the latest methods and techniques in contemporary language learning for more effective language teaching have attracted interest greatly. These tendencies of change in teaching/ learning pedagogy towards individual learning require individual guidance from the learner in their implementation.

The research implies that individual language learning counselling is used much more efficiently in Europe than in Turkey, as this is still a very young field of research in Turkey. Considered from this in order to be able to determine the importance of language learning advice more precisely and to explain the need for individual advice for language learners and language teachers in Turkey, a multi-faceted researcher/ consultant perspective turns into a twelve-week language learning counselling with a focus on writing, as part of a dissertation, an action research at the German teacher training of University Trakya in Edirne carried out.

The type of action research is based on observations, data collection, archiving of the implementation and its evaluations (cf. Johnson 2015). The data collections are recorded quantitatively and qualitatively. The reason for this approach is to support the effect of the writing counselling on the students in addition to qualitative research results, which will be based on analyses and probability interpretations, with quantitative data and thus to be able to show the final results as clearly as possible. The correlation of the two methods, so-called 'mixed methods' (also known as method triangulation or multi-method approach), do not constitute a conflict; on the contrary, a combination of qualitative and quantitative research can eliminate the weaknesses of both research methods (cf. Schneider 2014: 15; Kelle / Erzberger 2000: 299).

A precise process is used to collect, transcribe, analyse, evaluate and archive the data from a large amount of data. The students of all German teacher training departments of the universities in Turkey form the population of this research and students of the German teacher training of the University of Trakya from the academic year 2018-2019 define the sub-population, the top-down method of conscious or deliberate sampling is used in the sampling procedure. In this extended article, however, only a randomly selected individual case is presented.

The language counselling focused on writing, as the writing process of foreign language learners reveals an important aspect of language learning.

At the beginning of the writing counselling, existing experiences, the level of language proficiency and the individual strategy repertoire of the test person are recorded and evaluated. After an apprenticeship contract has been agreed, their deficit areas, upcoming curricula and learning objectives are jointly drawn up and the consultations are designed and carried out accordingly under these conditions. Furthermore, the test subjects are offered numerous documents, worksheets and training material relating to learner biographies, learning preferences, the promotion of strategy knowledge and text sample designs. This is intended to promote the awareness of one's own language competence and the acquisition of language awareness. The implementation was carried out according to a writing learning counselling model created by the counsellor (cf. Karaoğlu Bircan 2020: 187).

The research resulted in the test subject benefiting from the advice to learn to write and having removed many of her blockages and deficits in German writing. Furthermore, according 
to her own evaluations and the views of the consultant, the test person received further training in strategic procedures and autonomous work, and was able to transfer her profit shares outside of the consultation in other language learning areas. On the basis of this, the following suggestions are made: Individual language learning counselling should be established in Turkey both in secondary schools and in higher education. As this action research also showed, language learners can learn languages more effectively and develop their sensitivity and autonomy for language learning if they are given individual help. However, a prerequisite for the success of a counselling is the voluntariness of the person seeking advice. It is difficult to imagine that a person would make something out of a counselling, accept it, when he actually does not want it himself. That is why it is so important that those seeking advice make an appointment or a conversation themselves and are not sent by others.

Finally, it should be noted that effective consultations are designed professionally and should be designed according to precise preliminary planning. 


\section{Einleitung}

„Wenn man einem Menschen einen Fisch gibt, so ernährt man ihn für einen Tag, wenn man ihn lehrt zu fischen, so ernährt man ihn für sein Leben." Diese philosophische Ansicht von Konfuzius entspricht dem Grundgedanken dieser Forschung. Denn mit dieser Forschung ist es beabsichtigt den Lernern mittels einer Sprachlernberatung (demnächst: SLB) bei ihren Lernproblemen Wegweiser zu sein, sowie ihr Weiterlernen zu fördern. Die Auswahl für den Forschungsprozess SLB im Fokus Schreiben wird aus dem Grunde getroffen, da der Schreibprozess von Fremdsprachlernern einen wichtigen Aspekt beim Sprachlernen aufzeigt. Er bildet eine Voraussetzung für die ganze Sprachlernphase.

Diesbezüglich wird im Rahmen der Forschung an der Deutschlehrerausbildung (demnächst: DLA) der Universität Trakya in Edirne eine Schreiblernberatung (demnächst: SCHLB) konzipiert, problemartige Schreiblernsituation geschildert und mögliche Lösungswege aufgesucht. Bei dieser Forschung wird die Problemfrage, ob DaF-Lerner mit einer individuellen SLB ihren Schreibprozess fördern, untersucht. Die durchgeführte Aktionsforschung wird in dieser Studie an einem Fallbeispiel ausführlich dargelegt. Der Forschungsprozess wird ausführlich beschrieben, analysiert und bewertet. Abschließend werden aus den Forschungsresultaten abgeleitete Vorschläge angeführt.

\section{Forschungsüberblick}

Die Entwicklung der Lehr- und Lernkultur in der neuen Gesellschaft führt zu der Erkenntnis, dass sich der Mensch vielfältig entwickeln und belehren muss. Diese Realität bedingt eine vielseitige Führung in breiten Gebieten. Diese Führungen lassen sich unter den Begriffen 'Coaching' oder ,Beratung' einordnen. In Anbetracht dessen definieren Käpplinger / Rohs (2014) Lernberatung (demnächst: LB) als Antwort auf die Erwartungen der modernen Arbeitswelt und seine Lernherausforderungen und stellen die Etablierung der LB im Kontext lebenslangen Lernens in der Weiterbildung der Betriebswelt in den Vordergrund. Bei Siebert (2006) ist der Begriff LB eher die gemeinsame Schnittmenge in einer Vielfalt von Aspekten des selbstgesteuerten Lernens und Claußen (2009) sieht LB als ein Ideenpool für die Horizonterweiterung. Für Tok (2015) ist die Lernberatung eine Hinführung zur Autonomität. LB wird in ihrer Entwicklung von verschiedenen Ansätzen beeinflusst, sie umfasst ein weites Feld in der Erziehungswissenschaft sowie Psychologiewissenschaft und wird auch von „gesellschaftlichen Implikationen“ beeinflusst (vgl. Pätzold 2006: 1; Käpplinger / Rohns 2014: 24), die als kommerzielle Aspekte auftreten, wie z.B. als synonymer Begriff ,Coaching'. Jedoch zeigen die Definitionen der Autoren bei der Beschreibung des Begriffs LB Parallelitäten auf, insbesondere in der Realität, dass die Beratungen gewissermaßen einem Ratsuchenden Weg-Weiser sein sollen. Zufolge wird LB in dieser Studie aus der Perspektive eines Ratsuchenden nach der Auffassung von Kleppin betrachtet „dem Lernenden zu ermöglichen, zu einem Verständnis seiner selbst zu gelangen und auf Grund dieser neuen Orientierung positive Schritte zu unternehmen“ (Kleppin 2010: 1163).

In der Fremdsprachenlehre wird öfters für die LB auch der Begriff SLB 
verwendet. Desgleichen zielt die SLB laut Chromcak (2008) darauf ab, Lernende dabei $\mathrm{zu}$ unterstützen, in ihrem Fremdsprachenlernen für den eigenen Lernprozess Verantwortung zu übernehmen und effiziente Lernwege zu finden. „Sprachlernberatung ist eine kurzfristige soziale Interaktion zwischen Sprachlernberatungen und den Lernern einer fremden Sprache, bei der den Lernern eine Unterstützung bei der Lösung ihrer auf das Sprachlernen bezogenen Probleme angeboten wird“ (Chromcak 2008: 16). $\mathrm{Zu}$ dem bezeichnet Chromcak die individuelle durchgeführte Art der SLB, als die relevante Form, welche mit nur einem Lernenden pro Sitzung stattfindet (vgl. ebd.: 14). Saunders sieht in der -dem nichtdirektiven, personenzentrierten Beratungsansatz von Rogers angeknüpften- SLB eine Förderungsmöglichkeit der Lernerautonomie beim Fremdsprachenlernen.

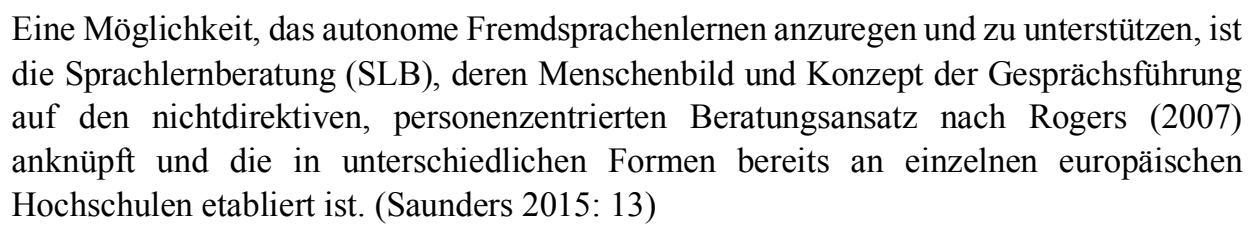

Eine Möglichkeit, das autonome Fremdsprachenlernen anzuregen und zu unterstützen, ist die Sprachlernberatung (SLB), deren Menschenbild und Konzept der Gesprächsführung auf den nichtdirektiven, personenzentrierten Beratungsansatz nach Rogers (2007) anknüpft und die in unterschiedlichen Formen bereits an einzelnen europäischen Hochschulen etabliert ist. (Saunders 2015: 13)

Die Beratung, die in den europäischen Regionen das Problem Schreiben und seine Förderung in den Fokus setzt, wird als SCHLB bezeichnet: „Unter Schreiblernberatung verstehe ich ein Eins-zu eins-Gespräch, bei der ein ausgebildeter Schreiblernberater Schreibende beim Verfassen eines Textes unterstützend begleitet. Besonders wichtig ist dabei, dass die Beratenden sich auf die Schreibenden und deren Bedürfnisse, Stärken und Schwächen einstellen.“ (Grieshammer vd. 2016: 13)

SLB als Forschungsgebiet weist im europäischen Kontext hochwertige, ideenreiche und auch praxisbezogene Literatur auf. Diesbezüglich kann die europäische Forschungsliteratur gegenüber der Forschungsliteratur im Inland als eine Bereicherung für die Forscher bzw. Forschungen angesehen werden. Der theoretische Rahmen der LB wird von vielen Autoren in Europa didaktisch, methodisch, modelhaft und aber auch kritisch vertreten. Insbesondere zum Fachdiskurs und Theorie der LB bzw. SLB können u.a. Fachstudien von Siebert (2006), Chromcak (2008), Vogler / Hoffmann (2011) angegeben werden. $\mathrm{Zu}$ modelhaften Studien können u.a. auf diese Studien hingewiesen werden: Grieshammer vd. (2016) beschreiben die Gesprächsführungsmethoden und Techniken unter Angaben persönlicher Erfahrungen und Fallanalysen, stellen auch somit eine Basisliteratur für diese Forschung dar. Saunders (2015) bevorzugt in ihrer Forschung die Online-SLB-Methode in Zusammenhang mit einer , face to face' Beratung und lehnt sich wie auch viele andere Forscher an Rogers (1992) ,nicht-direktiven, personenzentrierten Beratungsansatz'. Claußen (2009) bearbeitet in ihrer Studie LB mit Strategietraining.

Hingegen ist LB/ SLB in der Türkei noch ein ziemlich neues Feld, diesbezüglich bietet es wenig Forschungsliteratur an. Diese Gegebenheit zeigt unteranderem, dass hier ein Forschungsbedarf vorhanden ist. An dieser Stelle kann auf Tok (2015) referiert werden, die auf die Wichtigkeit der SLB eingeht und einen Ablaufprozess der SLB mit ihren Lernern darstellt. Die Studie von Köksal (2019) weist auf den neusten Forschungsstand der SLB, auf die SLB Tagungen in Italien, Potsdam, und Hannover hin, 
bei denen verschiedene Wissenschaftler der Sprachlehre die Konturen der SLB und Sprachlernberater bzw. SCHLB und Schreiblernberater diskutieren.

\section{Ziel und Fragestellung}

Das Ziel dieser Forschung besteht darin, eine institutionell eingerahmte, professionell konzipierte SLB mit Fokus Schreiben in der DLA der Universität Trakya durchzuführen. Dabei wird die Forschungsfrage gestellt, ob DaF-Lerner durch eine individuelle SLB ihren Schreibprozess fördern können.

\section{Methode}

Angelehnt an den bereichernden Beschreibungen u.a. der Forscher Ferrance (2000), Johnson (2015), Gürgür (2016) wird in dieser Studie eine Aktionsforschung durchgeführt. Die Forschung richtet sich mehr nach Unterteilung der Aktionsforschung von Ferrance (2000: 6) und wendet die ,Individuell Teacher Research Methode' ${ }^{3}$ an, wobei der Lehrende -hierbei auch der Beratende- an seinen Lernern Probleme erfasst und durch gezieltes planvolles methodisches Zusammenhandeln und einer ausführlichen Datensammlung diesen auf den Grund geht und effektive Lösungswege finden möchte.

Die SLB und SCHLB wird ausführlich dokumentiert, analysiert und evaluiert. Der ausgewählte Forschungsansatz erfolgt qualitativ und quantitativ. Die Korrelation beider Methoden sogenannte ,mixed methods', auch als Methodentriangulation oder MehrMethodenansatz bezeichnet, stellen keinen Konflikt dar, ganz im Gegenteil eine Kombination aus qualitativer und quantitativer Forschung kann die Schwächen beider Forschungsmethoden eliminieren (Kelle / Erzberger 2000: 299).

\section{Teilgesamtheit}

Die Studenten aller Deutschlehrerausbildungsabteilungen der Universitäten in der Türkei bilden die Grundgesamtheit dieser Forschung und Studenten der DLAA der Universität Trakya aus dem Studienjahr 2018-2019 definieren die Teilgesamtheit. Bei dem Stichprobeverfahren wird u.a. gemäß Schiffauer (vgl. Schiffauer vd. 2009) das TopDown-Verfahren der bewussten und absichtsvollen Stichprobenziehung angewendet. Für die Teilerhebung bzw. Stichprobe werden mit einem Flugblatt allen Studierenden der Universität Trakya DLAA 2017/ 2018 die Beratungsangebote mitgeteilt. Nach einer Bedürfnisanalyse und einer Textproduktion zu einer bestimmten Textsorte, worin Indizien für die Schreibhaltung erkennbar sind, wird die endgültige Probandenauswahl getroffen. Eine Studentin aus dem zweiten Semester verfasste als einzige freiwillig die Bedürfnisanalyse und die schriftlichen Textproduktionen in der deutschen Sprache, was auch zur Auswahl dieser Studentin als Probandin für diese Studie führte. Es wurde 12 Wochen lang 6-mal individuelle SCHLB von mindestens einer Stunde durchgeführt.

\footnotetext{
${ }^{3}$ Forscherübersetzung: individuelle Lehrerforschung
} 


\section{Datenerhebung und Analyse}

Bei der Datenerhebung wird u.a. von dem Mills Model (2011) (vgl. Gürgür 2016) profitiert. Dieses Modell ist ein Dialektisches-Zyklus-Modell, welches auf Bestimmung des Fokusbereiches, Datensammlung, Analyse und Auswertung der Daten, schließlich auf den Aktionsplan beruht. Der Fokusbereich bildet die SCHLB aus dem Konzept der SLB heraus, dementsprechend werden in den Beratungen vielfältige Daten gesammelt qualitativ und quantitativ ausgewertet, analysiert und evaluiert. Für die Behebung der Defizitbereiche werden Vorschläge für die Probandin geplant und weiterempfohlen, was als ein Aktionsplan angesehen werden kann. Dieses Vorgehen beruht in sich auf einem dialektischen Zyklus. Gemäß den Bedürfnissen wird in diesem Zyklus immer wieder unter denselben Kriterien gehandelt.

Die qualitativen Daten werden transkribiert, analysiert und evaluiert. Bei Bewertung der quantitativen Daten werden die ,textinternen Kriterien ' berücksichtigt. Die strukturelle/ grammatische Kohärenzebene und die Kohäsionsebene haben primäre, die thematische/ Inhaltsebene sekundäre Stellung. In dieser Forschung wird Kohäsion u.a. nach Vaters Beschreibung definiert, „[...] Kohäsion ist die grammatische Relation zwischen Einheiten des Textes. Sie tritt satzübergreifend auf" (Vater 2001: 31-66). Bei der Analyse und Bewertung der Daten angelehnt an Ferrance (2000) wird eine synchrone Auswertung der Daten durchgeführt. Die qualitativen Datensammlungen beruhen u. a. auf:

- Fragebogen zur Auswahl der Probandin,

- Individuelle Gespräche; Beobachtungen des Forschers; Video und Tonaufzeichnungen,

- Dokumente als Arbeitsmaterialien bei Strategietrainings und Korrekturen,

- Schriftliche Texte/ Aufgaben.

Universell gesicherte 17 verschiedene Daten ${ }^{4}$ werden in der SLB aus der oben zusammengefassten Daten-Palette verwendet. Dieses Arbeitsmaterialangebot (AM) wird von der Beraterin aus einem reichen Inventar ausgewählt u.a. aus Bimmel und Rampillon (2000), Ballweg vd. (2013), Funk vd. (2014), Barkowski vd. (2014). Die Auswahl basiert individuell auf Defizite, Erfordernisse und Lernziele der Probandin.

Die quantitativen Daten dieser Forschung erfolgten zum größten Teil aus Tests und Schreibaufgaben der Probandin, welche von der Beraterin nach einer präzisen Auswertung und Fehlerkorrektur in Tabellen aufgeführt sind. Die tabellarischen Fehleranalysen-Raster zu den rezipierten Texten werden von der Forscherin individuell gestaltet. In der SCHLB wurden als quantitative Daten von folgenden Daten profitiert:

\footnotetext{
${ }^{4}$ Details zu den qualitativen und quantitativen Daten sowie ihren Quellen werden in der Dissertationsarbeit von Karaoğlu Bircan unter Kapitel Teil 3.2.1.und 3.9 und im Anhangsteil aufgeführt (vgl. Karaoğlu Bircan 2020:170-171; 201-207).
} 
- Fragebogen Phase $\mathrm{D}^{5}$,

- EST/ A2 (Deutsch-Einstufungstest GER/ DT-GER A2) ${ }^{6}$,

- EST Schriftlicher Ausdruck (Einstufungstest Schriftlicher Ausdruck) ${ }^{7}$,

- EST/ B1 (Deutsch-Einstufungstest GER/ DT-GER B1)

- Fehleranalysen-Raster zu ausgewählten schriftlichen Aufgaben der Probandin.

Bei der Datenauswertung wird eine indirekte und gezielte Fehlerkorrektur vorgenommen. Ausgewählte Fehler werden im Text gekennzeichnet, ohne dass Verbesserungsvorschläge unterbreitet werden. Die Fehlerkorrektur bezieht sich auf Morphologie und strukturelle Syntax. Bei der Morphologie werden Verstöße gegen das Genus, Kongruenz in Nominalphrasen gekennzeichnet, wobei die Flexionsendungen der Artikelwörter, Pronomen berücksichtigt werden. Das Wort mit der falschen oder fehlenden Flexionsendung wird markiert. Zur Syntax werden Verstöße gegen Wortstellungsregeln gekennzeichnet. Der betroffene Haupt- oder Nebensatz wird unterstrichen. In der Fehlertypenkategorie ${ }^{9}$ wird angelehnt an Kleppin (2002) vorgegangen.

Die Auswahl der Beratungssprache in den Beratungen wird der Probandin überlassen. Beabsichtigt wird damit die Herstellung einer gegenseitigen und synchronen, direkten und verständnisvollen Kommunikation zwischen der Beraterin und der Probandin. Somit ergab es sich, dass die Beratungen zum größten Teil in der türkischen Sprache mitunter in einer Interimsprache (Deutsch-Türkisch) abliefen und folglich wurde eine besondere Form von Transkription erforderlich. Wie auch Claußen (2009) betont, bedarf es sehr viel Mühe und Aufwand alle Gesprächspassagen zu transkribieren, insbesondere bei Probanden mit unterschiedlichen Sprachkenntnissen. Viele Forscher haben in ihren Studien verschiedene Transkriptionsformen für Dokumentation der Beratungsgespräche aufgeführt (vgl. u.a. Claußen 2009: 109-112; Grieshammer vd. 2016: 94; Tressel 2017: 158). In dieser Forschung wird mehr von der Transkriptionsform von Claußen (ebd.) profitiert. Bei der Transkription werden nur Gesprächspassagen wörtlich zitiert, die für das Erkenntnisinteresse dieser Studie relevant sind. Nur für den Beratungsprozess bedingte Verhalten und Ausdrücke werden ausführlich in der Originalversion der angewandten Sprache (hierbei mehr Türkisch) zitiert, paraverbale Merkmale wie ,lächeln oder lachen usw.' und nonverbale Merkmale werden im Dialogtext zwischen Klammern in der deutschen Sprache erklärt. Die Gesprächspassagen werden unter den Transkriptionstexten in der Zielsprache Deutsch zusammengefasst. Bei

\footnotetext{
${ }^{5}$ Vgl. Karaoğlu 2020: 378

${ }^{6} \mathrm{https} / / / \mathrm{eb}-$ zuerich.ch/site/filemanager/files/pdf/einstufungstests/deutsch-a1.pdf

${ }^{7} \mathrm{https}$ ://www.klett-sprachen.de/download/828/berliner_platz_NEU_ET.pdf

${ }^{8} \mathrm{https}$ ://ebzuerich.ch/site/filemanager/files/pdf/einstufungstests/deutsch-a1.pdf

9 adaptierte Abkürzungen: Für falsche Rechtschreibung (Rs.); Zeichensetzung (Zs.); Flexion (Flx.); Artikel (Art.); Präposition (Präp.); Modalverb (Modv.); Tempus (Temp.); Satzstellung (Satzst.); Ausdruck (Aus.); Singularform (S); Pluralform (P); Pronomen (Pro.); falsches Wort (Wort) (vgl. Kleppin 2002: 45-47).
} 
der Transkription wird die Rechtschreibung, und Zeichensetzung in beiden Sprachen, hierbei Deutsch und Türkisch je nach der Anwendung beachtet, jedoch Aussprache und Strukturfehler werden nicht beachtet, dem Original nach wiedergegeben.

\section{Einzelfallanalyse}

Vorstellung der Probandin (P5) ${ }^{10}$ : Die folgenden Feststellungen aus der Tab.1 stammen aus den Antworten zu dem Fragebogen, den Selbsteinschätzungs-/ Lerntypen-/ Lernstiltests von P5 und den Beraternotizen.

P5 als eine Studentin für das Lehramt DaF im zweiten Semester erwartet von dem Beratungsprozess sich besser schriftlich ausdrücken $\mathrm{zu}$ können, sowie einige Schreibstrategien kennenzulernen, die ihr dabei helfen werden, sich u.a. auf das ErasmusProgramm, welches P5 nächstes Semester antreten soll, vorzubereiten. Ausführliche Angaben über die Probandin können aus der Tab.1 entnommen werden.

\begin{tabular}{|c|c|}
\hline Probandin & P5 \\
\hline Studium & DLAA, Universität-Trakya \\
\hline Semester/ & 2 (+ 2 Semester Vorbereitungsklasse) \\
\hline Durchschnitt & 3,75 (sehr gut) \\
\hline Herkunft/ Wohnort & Edirne/ Türkei; wohnt alleine in einem Apartment \\
\hline Alter/ Geschlecht & 22/ weiblich \\
\hline L0/ Niveau & Türkisch/ C2 (eigene Einstufung) \\
\hline L1/ Niveau & Englisch /A1 (nach eigener Einstufung) \\
\hline L2/ Niveau & $\begin{array}{l}\text { Deutsch A2 (nach eigener Einstufung), B1 (nach dem EST) } \\
\text { Für Deutsch-Schreiben nach dem Fragebogen-Text Stufe A2 ersichtlich. }\end{array}$ \\
\hline Besonderheiten & $\begin{array}{l}\text { Fragebogen wird auf Deutsch beantwortet } \\
\text { Keine Auslandserfahrung; plant Teilnahme an einem Erasmus- } \\
\text { Austauschprogramm (Potsdamer Universität-DLAA) hat die } \\
\text { Teilnahmeprüfung bestanden; zweifelt, ob ihre sprachlichen Kenntnisse } \\
\text { für ein Studium in Deutschland ausreichen. } \\
\text { Lebt und arbeitet ordentlich. } \\
\text { DT-GERA2 und DT-GERB1 positiv }\end{array}$ \\
\hline Lerntyp (Präferenzen) & Handelnder Lerner (Taster) \\
\hline Lernstil & Aktiver Experimentierer: Entscheider \\
\hline Hirndominanz & Rechts Dominant, eher kreativ tendiert \\
\hline Lernstrategie-Kenntnisse & Kein Schreibstrategiewissen \\
\hline
\end{tabular}

${ }^{10}$ Die Probandin wird mit dem Codezeichen P5 benannt, um die Identität (Anonymität) der Person zu schützen. 


\begin{tabular}{|l|l|}
\hline Autonomes Lernen (AL) & Geringe Kenntnisse über AL, lernt gerne regelmäßig, aktiv \\
\hline $\begin{array}{l}\text { Defizite im Bereich } \\
\text { Schreiben }\end{array}$ & $\begin{array}{l}\text { Unzureichende Schreib-Wortschatzkenntnisse } \\
\text { Schreibnutzung: Notizen machen, Hausaufgaben, Vorlesungen }\end{array}$ \\
\hline $\begin{array}{l}\text { Defizite in anderen } \\
\text { Bereichen }\end{array}$ & Fehlendes Sprachbewusstsein und Sprachverstand \\
\hline Starke Bereiche & $\begin{array}{l}\text { Schreibt gerne; plant und überarbeitet ihre Texte. } \\
\text { Kann Haupt- und Nebensätze bilden (Konjunktionengebrauch im Bereich } \\
\text { A2 und B1). } \\
\text { Kann korrekte Inversionen bilden/ gute Orthografie und Zeichensetzung }\end{array}$ \\
\hline Erwartungen & $\begin{array}{l}\text { Besserer Ausdruck und ausführliche Erzählweise } \\
\text { Förderung des Schreibens } \\
\text { Aneignung von Strategiekenntnissen }\end{array}$ \\
\hline
\end{tabular}

Tab. 1: Wesentliche Angaben und Informationen über P5 in Übersicht ${ }^{11}$

\section{Gesprächstranskriptionen und Bewertungen von P5}

In der ersten Beratung (B1) nach der Vorstellungsphase geht die Beraterin (B) auf das Thema Schreiben SCHLB und Schreiblernstrategien (SCHLS) ein, um die Gedanken und Vorwissen der Probandin herauszufinden. Dabei wird ersichtlich, dass P5 keine genauen Vorstellungen über Schreiben, SCHLB und SCHLS hat.

B1/ Teil1/ P5: 00.03:40 Min.
$[\ldots]$
B: Yani, sen kendi duygularından beklentilerinden düşüncelerinden biraz bahsedebilirsin?
P5: Ehem... Yazma konusunda kendimi yetersiz görüyorum. Ehem öncelikle yazma tekniği bilmiyorum.
B: (Nickt aufmunternd) Hihl...
P5: (Lächelt)Yazma tekniklerine dair hiçbir fikrim yok. Bazen kendimi ifade edemiyorum o yüzden hep böyle basite indirgemek zorunda kaliyorum Almanca anlatımımda. Bu biraz kelime bilgisi eksikliğinden de kaynaklaniyor bence...

B: A öyle mi düşünüyorsun?

P5: Evvet. Genelde yazma dersi için yazıyorum zaten. Normalin bi (stoppt)

B: Onun dişıinda yaz...?

P5:(unterbricht) Onun dışında hayır, yazmayla çok fazla uğraşmıyorum.

B: Öğrenim sürecinde yazma stratejileri konusunda hiç bilgi aldın ml, kendin araştırdın mi, veyahut bu konuda bir repertuarın oluşmuş mudur?

P5: (Schüttelt den Kopf) Yok. Hayır.

${ }^{11}$ Die Tab.1 sowie alle anderen Tabellen wurden von der Forscherin selbst erstellt. 
B: Nasıl çalışırsıı?

P5: Genel olarak mi?

B: Yani genel olarak ve yazma bunun içinde ne kadar yer altyor?

P5: Oturup bir metin yazmiyorum, normalde.

B: Metin oluşturma yapmıyorsun yani!

P5: Evet. Ehem... ama hep yazarak çalışıyorum. (Lacht) Kelimeleri yazarak çalışlyorum. Gramer, şeyleri onları cümle kurarak yapıyorum zaten.

B: Dilbilgisi kurallarını cümlelerin içerisinde kullanıyorsun öyle mi?

P5: Evet. Yeni öğrendiğim bağlaçlar, kelimleler bu tarz şeyleri genelde yazarak çalışıyorum.

[...] Sınav öncesi de yazarak çalışıyorum zaten. (Lacht) Çok fazla yazlyorum. Yani hep yazarak kaliyor benim aklımda.

P5 benutzt das Schreiben nur für ihren Sprachlernprozess als Mittel und glaubt mit Aufschreiben sich alles besser zu merken. P5 betont, dass sie sehr viel schreibt, jedoch sich als Lernziel das Schreiben nicht gesetzt hat. Das Strategiewissen fehlt ihr noch. P5 fühlt sich beim Schreibprozess unzureichend und denkt an ihre Defizite im Wortschatzbereich und die fehlenden Schreib-Techniken, welche Gründe für die geringe Ausdrucksfähigkeit sind. In diesem Zusammenhang werden mit P5 zahlreiche Schreibstrategieübungen durchgeführt.

Um bei P5 ein Bewusstsein für das Sprachlernen zu entwickeln wurde ein ausführliches Training im Bereich der Fehleridentifizierung durchgeführt. P5 zeigte Interesse und Fleiß bei der Erkundung der Fehler. Die Entdeckung Ihrer eigenen Fehler, der Versuch diese $\mathrm{zu}$ korrigieren und zu analysieren, erweckten bei P5 ein Selbstvertrauen, was in der sechsten Beratung (B6) deutlich hervortritt.

B6/ Teil1/ P5: 00.17:50 Min.

\section{$[\ldots]$}

B: Bu arada Fehlerkorrektur/Analyse yaptlk biraz.

P5:( Nickt eifrig). Evvet.

B: Bu da bir strateji eğitimimizdi. Diğer bir strateji eğitimimiz. Neydi Fehleranalyse? Neden önemliydi sence (fragt lächelnd)?

P5: Özellikle puanlama gibi şeylerde çok önemli olduğunu söylemiştik, hatanın neye bağlı olduğunu bilirsek en azından düzeltmesi de bir o kadar kolay olur. Bu da kişide bilinç oluşturan bir şey.

B: Aynen!

P5: Kendi hatalarinin farkina varllyor bu şekilde.

B: Çok iyi! Bilinç yani. Nedenini açıklayabiliyorsak bi kişinin neden o hata yoluna gitmiş acaba...

P5: (unterbricht) Çözüm yolu da bi o kadar açık oluyor.

B: Süper. Hataları da düzeltmenin bir yolu yöntemi oluyor. Mesela bunun için bize yardımcı ne vardı, hataları düzeltirken? 
P5: (Sucht etwas in ihren Unterlagen) Dosyamda var tam hatırlayamadım şimdi adını.

B: Ona ne diyebiliriz? Sen söyle bir isim.

P5: (Lächelt) Fehlerzeichen olabilir mi?

B: Güzel işte. Fehlerzeichen/System. bi sistem gerekiyor. Mesela neler var o sistemin içerisinde?

P5: Eee... Kisaltmalar var!

B. Evvet.[…]

Aus dem Strategietraining und den Feedback-Ansichten von P5 geht hervor, dass P5 die Relevanz der Fehleranalyse begriffen und die Anschauung gewonnen hat, dass das Bewusstwerden der Fehlerursache zu der Korrektur und letztendlich zur individuellen Lösung für den Sprachlerner führen kann. Mit intensivem Training im Rahmen der Vorbereitung auf das ein semesterlange Studium an einer deutschen Universität für das Erasmus-Projekt werden mit P5 die Kriterien für das wissenschaftliche Schreiben erarbeitet. U.a. werden die Besonderheiten einer ,Geschlechtsbewusstensprache ' in wissenschaftlichen Texten, die Redemittel für die Gliederungsteile eines wissenschaftlichen Textes und die Zitat-Regeln behandelt. P5 zeigt auch bei diesem Training eine beeindruckende Beteiligung.

B6/ Teil3/ P5: 00.05:50 Min.

\section{$[\ldots]$}

B: Geschlechtsbewusste Sprache bei wissenschaftichen Texten, bu konuda çalışmış̧tı dimi (zeigt auf das AM) sen bu konuda neler yaptın, sonra ne oldu, neler fark ettin?

P5: (Weist auf die Stellen auf ihrem Arbeitsblatt). Yaparken şunları fark ettim. Ehem böyle Lehrkraft, Lehrperson o tarz şeylerin kullanımı daha doğru olduğunu eğer cinsiyet belirtmeyceksem.

B: Yani cinsiyet ayrımı derken neyi kastetmis olduk, daha ayrıntılı söyleyecek olsak?

P5: Yazmada kadin ya da erkek belirtmemiz gerekmeyen yerlerde genel olarak konuşuyorsak şayet (zeigt auf die Redemittel die sie herausgearbeitet hat) bu tarz tanımlamalar daha doğru oluyor. [...]

B: Güzel. Aynen.

P5: (Unterbricht eifrig) Evvet, ben mesela yazılarımda erkek kullaniyordum hep daha öncesinde, bunun bilincine vardım. Artık bu konuya dikkat edeceğim.

B: Super. [...]

P5 vervollständigt die ihr überreichten AM mit Eifer und Disziplin. Sie recherchiert und notiert Redemittel für wissenschaftliches Schreiben und kann einige aufsagen. Weiter kann P5 die einfachen Zitate aufschreiben und in das Quellenverzeichnis eintragen. P5 lernt die Fachterminologie und eine geschlechtsbewusste Sprache kennen. Die Beraterin erklärt P5, dass sie an der deutschen Universität wissenschaftliche Texte erwarten würden, die sie lesen und möglicherweise darüber Hausarbeiten oder Semesteraufgaben verfassen muss und diese wiederum fachliche Schreibkenntnisse erfordern würden. 
Für die Verfassung eines wissenschaftlichen Textes muss P5 noch viel dazu lernen, jedoch P5 erreicht mit Hilfe der Beratungen das Bewusstsein einen wissenschaftlichen Text erkennen zu können bzw. die Gestaltungsmerkmale eines Fachtextes herauszuarbeiten. Diese Erkenntnisse können die ersten Schritte für die Förderung der fortgeschrittenen Schreibfertigkeit von P5 darstellen.

\section{Die Auswertung der qualitativen Daten von P5}

Für die Beratungen werden P5 verschiedene AM und Dokumente (D) ${ }^{6}{ }^{12}$ angeboten. In B1 werden P5 D zur Informationsbeschaffung über die SLB in einem Dossier ausgehändigt, mit dem Wunsch diese $\mathrm{D}$ zur nächsten Beratung durchzulesen. Vorerst werden die Resultate der Lern-Präferenzen laut den Tests (Kolb-Tests) ${ }^{13}$ besprochen und es wird klargestellt, dass die rechte Gehirnhälfte bei P5 dominant ist und in diesem Zusammenhang unbedingt das kreative Schreiben gefördert werden sollte. Diesbezüglich werden der Probandin AM für das ,Kreative Schreiben“ angeboten. Mit diesem AM wird P5 im Bereich des Kreativen Schreibens gefördert und verfasst erfolgreiche Texte nach der Strategie ,Stimulus Schreiben“ im Bereich ,Assoziatives Schreibens'. Ferner werden die Strategien des Notierens, Sammelns und Ordnens aus dem Bereich der ,Mind-Map Techniken' von P5 angewandt und positiv eingestuft. Für die Erweiterung und Festigung des Wortschatzes wird die Arbeit mit der Lernkartei und Satzgliederkartei vorgestellt. Diese werden von P5 relativ ernst aufgenommen und mit Begeisterung praktiziert. Mit der Probandin werden in allen Beratungen anhand ihrer Aufgaben Fehleranalysen durchgeführt. Die selbstständige Erkundung ihrer Fehler und der Ursachen sowie eigene Verbesserungsvorschläge stärken das Selbstvertrauen und Sprachbewusstsein von P5 und erhöhen die Lernmotivation.

Ein weiteres wichtiges Lernziel von P5 umfasste das formelle und fachliche Schreiben. Diesbezüglich werden die Textüberarbeitungskriterien im Bereich des argumentativen und fachlichen Schreibens, die Zitierformen und Quellenangaben und die Textgestaltungsmerkmale für das Schreiben eines Protokolls trainiert. P5 zeigte ein ähnlich großes Interesse und eine hohe Arbeitsperformanz mit formellen Texten, so wie auch mit den kreativen Texten.

Es kann festgehalten werden, dass P5 trotz ihrer belastenden Vorlesungen und ihrer Unsicherheiten hohe Erfolgstendenz und Lernmotivation aufzeigt. Die Beratungen waren bei der Behebung ihrer Unsicherheit beim Schreibprozess und bei ihren Struktur-/ Ausdruckdefiziten behilflich. Ihre Strategiekenntnisse wurden gefördert und ihr Selbstvertrauen gestärkt. Die Beratungen haben auch zum individuellen autonomen Lernen beigetragen. P5 muss nach den Beratungen individuell weiterarbeiten, was sie auch verwirklicht. P5 lernt hierbei auch, was und wie sie weiterlernen muss, wo und wie sie während des Erasmus-Programms an der Universität Potsdam Hilfe anfordern kann.

\footnotetext{
${ }^{12}$ Für detaillierte Informationen zum AM und D (vgl. Karaoğlu Bircan 2020: 267)

${ }^{13} \mathrm{https}$ //www.lernwerkstatt.ch/assets/kursunterlagen/sveb-kursleiter-lehren-und-lernen.pdf
} 


\section{Die Auswertung der quantitativen Daten von P5}

Die schriftlichen Texte von P5 aus dem Fragebogen und den EST werden von der Beraterin bewertet und in einem selbstgestalteten Tabellenraster als Übersicht zusammengetragen.

\begin{tabular}{|c|c|c|c|c|c|c|c|c|c|c|c|c|c|}
\hline \multicolumn{14}{|c|}{ Angaben zur Bewertung der Schreibaufgabe Text 1} \\
\hline $\begin{array}{l}\text { Probandi } \\
n\end{array}$ & \multicolumn{2}{|c|}{ Textsorte } & \multicolumn{2}{|c|}{$\begin{array}{l}\text { Anzahl } \\
\text { der Wörter }\end{array}$} & $\begin{array}{l}\text { Anza } \\
\text { hl } \\
\text { der } \\
\text { Sätze }\end{array}$ & \multicolumn{3}{|c|}{ Satzarten } & \multicolumn{2}{|c|}{ Anzahl } & \multicolumn{2}{|c|}{ Konjunktion } & Anzahl \\
\hline P5 & \multicolumn{2}{|c|}{$\begin{array}{l}\text { Tagesablauf } \\
\text { (aus dem } \\
\text { Fragebogen) }\end{array}$} & \multicolumn{2}{|l|}{79} & 10 & \multicolumn{3}{|c|}{$\begin{array}{l}\text { Anredesatz: } \\
\text { Aussagesatz: } \\
\text { Aufforderungssatz: } \\
\text { Fragesatz: } \\
\text { Begrüßungssatz: } \\
\text { Absender: }\end{array}$} & \multicolumn{2}{|c|}{$\begin{array}{l}1 \text { korrekt } \\
9 \\
--- \\
--- \\
---\end{array}$} & \multicolumn{2}{|c|}{$\begin{array}{l}\text { und: } \\
\text { um...zu: } \\
\text { deshalb: } \\
\text { nachdem: } \\
\text { damit: } \\
\text { bevor: }\end{array}$} & $\begin{array}{l}1 \\
1 \\
1 \\
1 \\
1 \\
1 \\
\text { Total: } 6\end{array}$ \\
\hline $\begin{array}{l}\text { (Text 1) } \\
\text { Fehler: } \\
11\end{array}$ & $\begin{array}{l}\text { Rs.: } \\
4\end{array}$ & $\begin{array}{l}\text { Zs.: } \\
---\end{array}$ & $\begin{array}{l}\text { Flx } \\
2\end{array}$ & $\begin{array}{l}\text { Art } \\
: \\
---\end{array}$ & $\begin{array}{l}\text { Präp: } \\
1\end{array}$ & $\begin{array}{l}\text { Mod } \\
\text { v: } \\
---\end{array}$ & $\begin{array}{l}\text { Tem } \\
\text { p.: } \\
1\end{array}$ & $\begin{array}{l}\text { Satz } \\
\text { st. } \\
---\end{array}$ & $\begin{array}{l}\text { Wort: } \\
1\end{array}$ & $\begin{array}{l}\mathrm{Au} \\
\mathrm{s}: \\
2\end{array}$ & $\begin{array}{l}\text { Pro.: } \\
---\end{array}$ & $\begin{array}{l}\text { S/P: } \\
---\end{array}$ & $\begin{array}{c}\text { Kohärenz } \\
\text { Korrekt }\end{array}$ \\
\hline
\end{tabular}

Tab. 2: Übersicht der Fehleranalyse zum Text 1 von P5

In der Schreibaufgabe Text 1 von P5 aus dem Fragebogen Phase-D können aus der GERA2-Ebene Wörter sowie Wörtergruppen verwendet werden. P5 hat in einem Alltagsablauf bestehend aus 10 Sätzen und 79 Wörtern insgesamt 11 Fehler gemacht. Je 2 Fehler betreffen die Bereiche der Flexion und des Ausdrucks, je 1 Fehler werden im Bereich Präpositionen, Tempus- und Wortwahl verzeichnet. 4 Fehler sind im Bereich Rechtschreibung zu vermerken. P5 hat 6 Konjunktionen verwendet, die der Niveaustufe A2 zugeordnet werden können. Bei der Textkohärenz ist kein Defizit vorhanden. Die Anwendung von 4 Nebensätzen und 3 korrekten Inversionsätzen weisen auf den Anfang der Stufe B1 hin. Jedoch die 2 einfachen Flexions- sowie 4 Rechtschreibfehler und die Ausdruckswahl könnten bei der Einstufung B1 ein Hindernis darstellen. P5 sieht ihre ,Alltagsablaufbeschreibung' als ein Tagebucheintrag an, was als korrekt aufgefasst werden könnte, jedoch die unpersönliche und sachliche Erzählperspektive kann diesbezüglich Bedenken hervorrufen. Diese Tatsache stellt ein Texteinstufungsproblem dar. 


\begin{tabular}{|c|c|c|c|c|c|c|c|c|c|c|c|c|c|}
\hline \multicolumn{14}{|c|}{ Angaben zur Bewertung der Schreibaufgabe Text 2 und Text 3} \\
\hline Probandin & \multicolumn{2}{|c|}{ Textart } & \multicolumn{2}{|c|}{$\begin{array}{l}\text { Anzahl } \\
\text { der Wörter }\end{array}$} & $\begin{array}{l}\text { Anzahl } \\
\text { der Sätze }\end{array}$ & \multicolumn{3}{|c|}{ Satzarten } & Anzahl & \multicolumn{2}{|c|}{ Konjunktion } & \multicolumn{2}{|c|}{ Anzahl } \\
\hline $\mathbf{P 5}$ & \multicolumn{2}{|c|}{$\begin{array}{l}\text { Text } 2 \\
\text { Persönliche/ } \\
\text { informelle E- } \\
\text { Mail }\end{array}$} & \multicolumn{2}{|l|}{55} & 7 & \multicolumn{3}{|c|}{$\begin{array}{l}\text { Anredesatz: } \\
\text { Aussagesatz: } \\
\text { Aufforderungssatz: } \\
\text { Fragesatz } \\
\text { Begrüßungssatz: } \\
\text { Absender }\end{array}$} & $\begin{array}{l}1 \text { korrekt } \\
4 \\
-- \\
1 \\
1 \\
\text { Vorname }\end{array}$ & \multicolumn{2}{|c|}{$\begin{array}{l}\text { weil: } \\
\text { wenn: }\end{array}$} & \multicolumn{2}{|c|}{$\begin{array}{l}1 \\
1 \\
\text { Total: } 2\end{array}$} \\
\hline P5 & \multicolumn{2}{|c|}{$\begin{array}{l}\text { Text } 3 \\
\text { Persönliche/ } \\
\text { Informelle E- } \\
\text { Mail }\end{array}$} & \multicolumn{2}{|l|}{68} & 9 & \multicolumn{3}{|c|}{$\begin{array}{l}\text { Anredesatz: } \\
\text { Aussagesatz: } \\
\text { Aufforderungssatz: } \\
\text { Fragesatz } \\
\text { Begrüßungssatz: } \\
\text { Absender }\end{array}$} & $\begin{array}{l}1 \\
6 \\
-- \\
-- \\
2 \\
\text { Vorname }\end{array}$ & \multicolumn{2}{|c|}{$\begin{array}{l}\text { dass: } \\
\text { aber: } \\
\text { um ... zu: }\end{array}$} & \multicolumn{2}{|c|}{$\begin{array}{l}1 \\
1 \\
1 \\
\text { Total: } 3\end{array}$} \\
\hline $\begin{array}{l}\text { (Text 2) } \\
\text { Fehler: } 1\end{array}$ & $\begin{array}{l}\text { Rs.: } \\
---\end{array}$ & $\begin{array}{l}\text { Zs.: } \\
---\end{array}$ & $\begin{array}{c}\text { Flx.: } \\
---\end{array}$ & $\begin{array}{l}\text { Art.: } \\
---\end{array}$ & $\begin{array}{c}\text { Präp.: } \\
---\end{array}$ & $\begin{array}{c}\text { Modv } \\
.: \\
---\end{array}$ & $\begin{array}{c}\text { Temp.: } \\
---\end{array}$ & $\begin{array}{c}\text { Satzs } \\
\text { t.: } \\
--\end{array}$ & $\begin{array}{l}\text { Wort: } \\
---\end{array}$ & $\begin{array}{l}\text { Aus } \\
\therefore\end{array}$ & $\begin{array}{c}\text { Pron. } \\
\text { : } \\
1\end{array}$ & $\begin{array}{l}\text { S/P: } \\
---\end{array}$ & $\begin{array}{c}\text { Kohären } \\
\text { z } \\
\text { (korrekt) }\end{array}$ \\
\hline $\begin{array}{l}\text { Text 3) } \\
\text { Fehler: } \\
1\end{array}$ & --- & --- & --- & --- & --- & --- & --- & 1 & --- & --- & --- & --- & (korrekt) \\
\hline
\end{tabular}

Tab. 3: Übersicht der Fehleranalyse zum Text 2 und Text 3 von P5

In der Tab. 3 sind die Ergebnisse der Fehlerkorrektur der Schreibtexte der persönlichen/ informellen E-Mails aus B1 (Text 2) und aus B6 (Text 3) von P5 vergleichend aufgeführt. Dementsprechend ist eine Übereinstimmung der totalen Fehleranzahl von einem (1) Fehler verzeichnet. Die eine (1) Fehlerdifferenz sortiert sich im Feld der Pronomen und Satzstellung. Die Wörteranzahl hat sich von (55) auf (68) gesteigert. Beim Gebrauch der Satzarten/ -anzahl ist eine Steigerung von 2 Sätzen feststellbar. Beim Gebrauch der Konjunktionsarten und -anzahl in beiden Textverfassungen sind keine signifikanten Veränderungen festzustellen. Die Text-Kohärenz in beiden Texten ist korrekt, d.h. P5 hat die Textstrukturbedingtheit der Schreibaufgabe gemäß den Anweisungen in zwei Verfassungen beachtet. 


\begin{tabular}{|c|c|c|c|c|c|c|c|c|c|c|c|c|c|}
\hline \multicolumn{14}{|c|}{ Angaben zur Bewertung der Schreibaufgabe Text 4 und Text 5} \\
\hline $\begin{array}{l}\text { Proband } \\
\text { in }\end{array}$ & \multicolumn{2}{|c|}{ Textart } & \multicolumn{2}{|c|}{$\begin{array}{l}\text { Anzahl } \\
\text { der Wörter }\end{array}$} & $\begin{array}{l}\text { Anzahl } \\
\text { der } \\
\text { Sätze }\end{array}$ & \multicolumn{3}{|c|}{ Satzarten } & Anzahl & $\begin{array}{l}\text { Konjunkt } \\
\text { ion }\end{array}$ & \multicolumn{3}{|c|}{ Anzahl } \\
\hline P5 & \multicolumn{2}{|c|}{$\begin{array}{l}\text { Text } 4 \\
S M S\end{array}$} & \multicolumn{2}{|l|}{31} & 5 & \multicolumn{3}{|c|}{$\begin{array}{l}\text { Anredesatz: } \\
\text { Aussagesatz: } \\
\text { Aufforderungssatz: } \\
\text { Fragesatz } \\
\text { Begrüßungssatz: } \\
\text { Absender }\end{array}$} & $\begin{array}{l}1 \text { kor. } \\
3 \\
-- \\
1 \\
--- \\
---\end{array}$ & da: & \multicolumn{3}{|c|}{$\begin{array}{l}1 \\
\text { Total: } 1\end{array}$} \\
\hline P5 & \multicolumn{2}{|c|}{$\begin{array}{l}\text { Text } 5 \\
\text { SMS } \\
\text { (aus B6) }\end{array}$} & \multicolumn{2}{|l|}{23} & 4 & \multicolumn{3}{|c|}{$\begin{array}{l}\text { Anredesatz: } \\
\text { Aussagesatz: } \\
\text { Aufforderungssatz: } \\
\text { Fragesatz } \\
\text { Begrüßungssatz: } \\
\text { Absender }\end{array}$} & $\begin{array}{l}--- \\
2 \\
1 \\
1 \\
-- \\
---\end{array}$ & $\begin{array}{l}\text { deswege } \\
\mathrm{n}\end{array}$ & \multicolumn{3}{|c|}{$\begin{array}{l}1 \\
\text { Total: } 1\end{array}$} \\
\hline $\begin{array}{l}\text { (Text 4) } \\
\text { Fehler: } \\
3\end{array}$ & $\begin{array}{l}\text { Rs.: } \\
---\end{array}$ & $\begin{array}{l}\text { Zs.: } \\
1\end{array}$ & $\begin{array}{l}\text { Flx.: } \\
1\end{array}$ & $\begin{array}{l}\mathrm{Ar} \\
\mathrm{t} .: \\
---\end{array}$ & $\begin{array}{l}\text { Präp.: } \\
---\end{array}$ & $\begin{array}{l}\text { Mod } \\
\mathrm{v} \\
---\end{array}$ & $\begin{array}{l}\text { Tem } \\
\text { p.: } \\
---\end{array}$ & $\begin{array}{l}\text { Satzs } \\
\text { t.: } \\
---\end{array}$ & $\begin{array}{l}\text { Wort: } \\
---\end{array}$ & $\begin{array}{l}\text { Aus.: } \\
\text {--- }\end{array}$ & $\begin{array}{l}\text { Pr } \\
\text { o.: } \\
---\end{array}$ & $\begin{array}{l}\mathrm{S} / \mathrm{P} \\
: \\
1\end{array}$ & $\begin{array}{l}\text { Kohären } \\
\mathrm{z} \\
\text { (korrekt) }\end{array}$ \\
\hline $\begin{array}{l}\text { (Text 5) } \\
\text { Fehler: } \\
\text { 0 }\end{array}$ & --- & --- & --- & --- & --- & --- & --- & --- & --- & --- & --- & --- & (korrekt) \\
\hline
\end{tabular}

Tab. 4: Übersicht der Fehleranalyse zum Text 4 und Text 5 von P5

In der Tab. 4 sind die Ergebnisse der Fehlerkorrektur der Schreibtexte der SMS/ kurze Mitteilung aus B1 (Text 4) und aus B6 (Text 5) von P5 vergleichend aufgeführt. Dementsprechend ist eine Reduzierung der totalen Fehleranzahl von drei (3) Fehlern auf null (0) verzeichnet. Die Fehlerreduzierungen sortieren sich im Feld der Flexion (1), der Zeichensetzung (1) und der Pluralform (1). Die Wörteranzahl hat sich von (31) auf (23) reduziert. Beim Gebrauch der Satzarten und -anzahl ist eine Reduzierung von einem Satz feststellbar. Beim Gebrauch der Konjunktionsarten und deren Anzahl in beiden Textverfassungen sind keine signifikanten Veränderungen festzustellen. Die TextKohärenz in beiden Texten ist korrekt, d.h. P5 hat die Textstrukturbedingtheit der Schreibaufgabe gemäß den Anweisungen in zwei Verfassungen beachtet. 


\begin{tabular}{|c|c|c|c|c|c|c|c|c|c|c|c|c|c|}
\hline $\begin{array}{l}\text { Proban } \\
\text { din }\end{array}$ & Textart & & $\begin{array}{l}\text { Anzahl } \\
\text { der } \\
\text { Wörter }\end{array}$ & \multicolumn{2}{|c|}{$\begin{array}{l}\text { Anzahl der } \\
\text { Sätze }\end{array}$} & \multicolumn{2}{|c|}{ Satzarten } & \multicolumn{2}{|c|}{ Anzahl } & \multicolumn{2}{|c|}{ Konjunktion } & & Anzahl \\
\hline P5 & $\begin{array}{l}\text { Text } 6 \\
\text { Forme } \\
\text { E-Mail } \\
\\
\text { Laus B. }\end{array}$ & & 29 & \multicolumn{2}{|l|}{5} & \multicolumn{2}{|c|}{$\begin{array}{l}\text { Anredesatz: } \\
\text { Aussagesatz: } \\
\text { Aufforderungssatz: } \\
\text { Fragesatz: } \\
\text { Begrüßungssatz: } \\
\text { Absender: }\end{array}$} & \multicolumn{2}{|c|}{$\begin{array}{l}1 \text { (korrekt) } \\
2 \\
--- \\
1 \\
1 \\
\text { Vorname }\end{array}$} & \multicolumn{2}{|l|}{ da: } & \multicolumn{2}{|r|}{ Total: 1} \\
\hline P5 & \multicolumn{2}{|c|}{$\begin{array}{l}\text { Text } 7 \\
\text { Formelle } \\
\text { E-Mail }\end{array}$} & 45 & \multicolumn{2}{|l|}{6} & \multicolumn{2}{|c|}{$\begin{array}{l}\text { Anredesatz: } \\
\text { Aussagesatz: } \\
\text { Aufforderungssatz: } \\
\text { Fragesatz: } \\
\text { Begrüßungssatz: } \\
\text { Absender: }\end{array}$} & \multicolumn{2}{|c|}{$\begin{array}{l}1 \text { (korrekt) } \\
3 \\
--- \\
--- \\
2 \\
\text { Vor/ und } \\
\text { Nachname }\end{array}$} & \multicolumn{2}{|c|}{$\begin{array}{l}\text { dass: } \\
\text { wegen: }\end{array}$} & \multicolumn{2}{|c|}{\begin{tabular}{|l|l}
1 \\
1 \\
\\
Total: 2
\end{tabular}} \\
\hline $\begin{array}{l}\text { (Text 6) } \\
\text { Fehler: } \\
5\end{array}$ & $\begin{array}{l}\text { Rs. } \\
: \\
---\end{array}$ & $\begin{array}{l}\text { Zs. } \\
: \\
1\end{array}$ & $\begin{array}{l}\text { Flx.: } \\
---\end{array}$ & $\begin{array}{l}\text { Art.: } \\
---\end{array}$ & $\begin{array}{l}\text { Präp.: } \\
---\end{array}$ & $\begin{array}{l}\text { Modv. } \\
1\end{array}$ & $\begin{array}{l}\text { Temp. } \\
: \\
1\end{array}$ & $\begin{array}{l}\text { Satzs } \\
\text { t. } \\
1\end{array}$ & $\begin{array}{l}\text { Wort: } \\
---\end{array}$ & $\begin{array}{l}\text { Aus: } \\
---\end{array}$ & $\begin{array}{l}\text { Pro. } \\
---\end{array}$ & $\begin{array}{l}\mathrm{S} / \mathrm{P} \\
\vdots \\
1\end{array}$ & $\begin{array}{l}\text { Kohärenz } \\
\text { (korrekt) }\end{array}$ \\
\hline $\begin{array}{l}\text { (Text } \\
7) \\
\text { Fehler: } \\
1\end{array}$ & --- & -- & --- & -- & -- & --- & --- & 1 & -- & --- & --- & -- & (korrekt) \\
\hline
\end{tabular}

Tab. 5: Übersicht der Fehleranalyse zum Text 6 und Text 7 von P5

In der Tab. 5 sind die Ergebnisse der Fehlerkorrektur der Schreibtexte ,Formelle -Mail' aus B1 (Text 6) und aus B6 (Text 7) von P5 vergleichend aufgeführt. Dementsprechend ist eine Reduzierung der totalen Fehleranzahl von fünf (5) Fehlern auf eins (1) verzeichnet. Die Fehlerreduzierungen sortiert sich im Feld der Zeichensetzung (1), der Modalverben (1), der Tempusform (1), der Satzstellung (1) und der Pluralform (1). Die Wörteranzahl hat sich von (29) auf (45) gesteigert. Beim Gebrauch der Satzarten/ -anzahl ist eine Steigerung von einem Satz feststellbar. Beim Gebrauch der Konjunktionsarten/anzahl in beiden Textverfassungen sind keine signifikanten Veränderungen festzustellen. Die Text-Kohärenz in beiden Texten ist korrekt, d.h. P5 beachtet die Textstrukturbedingtheit der Schreibaufgabe gemäß den Anweisungen in zwei Verfassungen.

Zwischenbilanz: Nach den ersten Werten der schriftlichen Aufgabe Text 1 (Tab. 2) liegt das Niveau von P5 beim Schreiben nach GER auf Niveau A2. Nach den Bewertungen EST- Schriftlicher Ausdruck (Tab. 3-5), die während B1 und B6 durchgeführt wurden, liegt das Niveau von P5 beim Schreiben deutschsprachiger Texte bei Niveau A2/ B1. Die Tabellenraster zeigen, dass P5 bei den Textproduktionsaufgaben 
in der B6 signifikant wenige Fehler aufweist. Bei Text 2 wird 1 Fehler, bei Text 3 kein Fehler, bei Text 4 nur 3 Fehler, bei Text 5 keine Fehler, bei Text 6 nur 5 Fehler, bei Text 7 nur 1 Fehler aufgezeichnet. Eine Fehlerreduzierung ist bei allen Textarten, wenn auch nicht prägnant, feststellbar. P5 äußert sich in den Schreibaufgaben aus der B6 ausdrücklicher und korrekter als aus der B1 und dem Text 1. Die festgestellten offensichtlichen Problembereiche von P5 die Textgestaltung, der Gebrauch von Flexionen, Zeichensetzung und Satzstellung haben sich verbessert. Demzufolge kann das für P5 angebotene Trainingsmaterial in den Beratungen als ein positiver Faktor eingestuft werden.

\section{Übersicht zur generellen Evaluation von P5}

Die unten von der Forscherin als Übersicht gestaltete Tab. 6 soll den Beratungsprozesses von P5 zusammenfassend darstellen und als ein Endergebnis fungieren. Die Aktivitäten, schriftlichen Dokumente sowie Video- und Tonaufnahmen aus den Beratungen werden teils aufgelistet (vgl. Karaoğlu Bircan 2020), teils archiviert.

\begin{tabular}{|c|c|}
\hline $\begin{array}{l}\text { Angewandtes } \\
\text { Trainingsmaterial }\end{array}$ & D13-16, D19-24, D27-38, D43-49 \\
\hline $\begin{array}{l}\text { SCHLS/LS/ } \\
\text { Lerntechniken } \\
\text { Aneignung }\end{array}$ & $\begin{array}{l}\text { Kreative Schreibtechniken: Freies Schreiben, Autobiografisches } \\
\text { Schreiben, Assoziatives Schreiben, Mind-Map und Cluster Technik } \\
\text { Verfassen von persönlichen und offiziellen E-Mails } \\
\text { Textüberarbeitungskriterien } \\
\text { Fehleranalysetechniken } \\
\text { Textmustertechniken von Protokoll und schriftlichen Referats } \\
\text { Formelles Schreiben (Zitieren, Fußnote, Quellenverzeichnis) } \\
\text { Individuelles und autonomes Lernen }\end{array}$ \\
\hline Tabellen- Resultate & $\begin{array}{l}\text { Fehlerreduzierung } \\
\text { Erkennbare Verbesserung: Textstruktur Flexionen, Präpositionen, } \\
\text { Pronomen, Orthographie; Zeichensetzung, Tempus, Satzstellung (vgl. Tab. } \\
\text { 3-5) } \\
\text { Textproduktion: Textmuster, Textgewebe; Textform, inhaltlicher Ausdruck } \\
\text { (vgl. Tab. 2-5) } \\
\text { Niveauanstieg Schreibfertigkeit: von A2 zu B1 } \\
\text { (vgl. Tab. 1-5) }\end{array}$ \\
\hline $\begin{array}{l}\text { Nachfrage der } \\
\text { Beraterin für eine } \\
\text { allgemeine Bewertung } \\
\text { der SCHLB. } \\
\text { Transkription: }\end{array}$ & $\begin{array}{l}\text { Mündliche Evaluation des Probanden zu der SCHLB } \\
\text { Transkription: B6/Teil3/ P5: 00:02Min. } \\
\text { P5: Ya benim için oldukça faydalı olduğunu düşünüyorum. Ehem... yazmayı } \\
\text { çok seven bir insanım. Özellikle yazma işinden çok keyif alıyorum ama } \\
\text { bugüne kadar duymadığım yazma stratejileri ve yazma teknikleri vs. gibi }\end{array}$ \\
\hline
\end{tabular}




\begin{tabular}{|l|l|}
\hline $\begin{array}{l}\text { B6/Teil3/P5: 00:01 Min. } \\
\text { B: [...] Ne düs̆̈̈̈nüyorsun } \\
\text { bu Beratunglarla ilgili } \\
\text { olarak? }\end{array}$ & $\begin{array}{l}\text { şeyler öğrenince, ögrendikten sonra daha doğrusu daha çok zevk almaya } \\
\text { başladım. En azından bazı şeylerin farkında olarak, bilincinde olarak, } \\
\text { bilinçli olarak yazmaya devam ettim. [...] Onun dışında yalnızca yazma } \\
\text { değil, yazma stratejileri, yazma teknikleri değil, özerk öğrenme konusunda } \\
\text { epey faydalı oldu. Açıç̧ası ben bu çallş̧manın içerisinde olduğum için çok } \\
\text { memnunum. }\end{array}$ \\
\hline $\begin{array}{l}\text { Berater-Exkurs als } \\
\text { allgemeine Auswertung }\end{array}$ & $\begin{array}{l}\text { P5 hat Strategieerkenntnis, Schreib-Motivation und Bewusstsein über } \\
\text { autonomes und individuelles Lernen gewonnen, Schreibangst-/Hemmungen } \\
\text { abgebaut; Selbstverstrauen gestärkt; Grammatik-/ und } \\
\text { Wortschatzkenntnisse erweitert. Die schriftlichen Texte haben sich } \\
\text { signifikant in Kohärenz und Kohäsion verbessert. Die Beratungen haben bei } \\
\text { der Orientierung und Studium im Erasmus-Programm an der Potsdamer } \\
\text { Universität relativ großen Beitrag geleistet, was in einer auf Deutsch } \\
\text { verfassten langen nachträglichen E-Mail aus Potsdam offensichtlich } \\
\text { ersichtlich ist (vgl. Karaoğlu Bircan 2020 Anhang 66-70). }\end{array}$ \\
\hline
\end{tabular}

Tab. 6: Übersicht der generellen Bewertung und Evaluation von P5

Entsprechend der mündlichen Evaluation von P5 kann festgehalten werden, dass P5 mit Hilfe der Beratungen bewusster und autonomer handelt, ihr Selbstvertrauen beim Sprachgebrauch entwickelt; ihre Fehler identifizieren kann; viele Schreibstrategien und Wortschatzerweiterungstechniken kennengelernt hat. Aus den schriftlichen Aussagen zu Evaluation der SCHLB wird die Motivation zum Schreiben und das Bewusstwerden beim Schreibprozess sowie die Aneignung effektiver Strategiekenntnisse erkenntlich (vgl. dazu Transkription B6/ Teil3/ P5: 00:02 Min.). In ihren schriftlichen Evaluationen äußert sich P5 ziemlich positiv zu der SCHLB, die Probandin fühlt sich nach den Beratungen sicherer und bewusster beim Schreiben. P5 findet das angewandte AM individuell auf sich bezogen und sehr hilfreich.

\section{Schluss und Vorschläge}

Als Schlussfolgerung zu dieser Forschung kann festgehalten werden, dass die Probandin mit der individuellen SCHLB gemäß den quantitativen Auswertungen (vgl. Tabelle 5 und 6) und den qualitativen Bewertungen $\mathrm{zu}$ den schriftlichen Arbeiten in ihrem Schreibprozess weitergefördert wurde. Zudem belegen die Evaluationen der Probandin aus den Beratungen, dass die SCHLB als Fortschritt für das Schreiben und die Schreibstrategienkenntnisse wahrgenommen wurde, ferner als hilfreich, motivierend, wirksam, letztendlich als Sprachbewusstheit und Autonomie fördernd vermerkt wurde. Jedoch sollte unbedingt beachtet werden, dass die Voraussetzung für das Gelingen einer Beratung die Freiwilligkeit des Ratsuchenden ist. ,Man kann ein Pferd zwar zum Brunnen führen - trinken machen kann man es jedoch nicht' mit diesem alten Sprichwort ist ein wesentliches Merkmal von Beratung angesprochen. Deswegen ist es auch so erheblich, dass die Ratsuchenden selber einen Termin bzw. ein Gespräch vereinbaren wollen.

Anhand der Forschungsergebnisse dieser Studie könnten folgende Vorschläge bereichernd für die weiteren Forschungen und Umsetzungen sein:

- Die Diskussionen um ein Modul für die Umsetzung der SLB in den verschiedenen Institutionen und Schulen sollten gefördert werden. Jedoch 
sollte dabei unbedingt beachtet werden, dass präzise durchdachte und detailliert vorgeplante Beratungen effektive Resultate hervorbringen können. Somit wäre es empfehlenswert, nach einem Beratungsmodell vorzugehen. In gleicher Weise wurde in dieser Forschung nach einem von der Forscherin im Rahmen einer Dissertationsarbeit für Umsetzungen einer SCHLB gestalteten Schreiblernberatungsmodell (vgl. Karaoğlu Bircan 2020: 187) vorgegangen. Bei der Erstellung dieses Modelles wurde u.a. von den Studien von Grieshammer vd. (2016: 131), von dem LBModell von Pätzhold (2006: 20) sowie von dem selbstgestalteten „Schreibprozessmodell“ der Autoren Lubzyk / Kielmann / Haas (2016: 158) im Rahmen eines Integrativ-Projekts des Nürtinger Beratungsmodells IBIS zur Förderung des akademischen Schreibens an der HfWU profitiert.

- Ansätze bezüglich der Ausbildung der Tutoren/ Berater/ Mentoren sollten sich in dem Curriculum der Erziehungswissenschaftlichen Fakultäten in der Türkei etablieren. Zu diesem Zweck wurde von der Forscherin ein Modell-Vorschlag ,Fortbildungskonzept SLB-DaF“ (vgl. Karaoğlu Bircan 2020: 356-358) betreffs der Ausbildung der Deutschlehrerkanditen der DLA-Universität Trakya als eine Sensibilisierung zum Erlangen eines Vorwissens über Sprachlernberatung erstellt.

- Weitere Studien über SLB in Fokus anderer Fertigkeiten könnten den Ratsuchenden weiterhelfen und für die Umsetzung der SLB bereichernd sein.

\section{Literaturverzeichnis}

Ballweg, Sandra u.a. (2013): Wie lernt man die Fremdsprache Deutsch? München: Goethe Institut, KlettLangenscheidt.

Barkowski, Hans u.a. (2014): Deutsch als Fremdsprache. Deutsch Lehren Lernen. München: Klett, Langenscheidt, Goethe Institut.

Bimmel, Peter / Rampillon, Ute (2000): Lernerautonomie und Lernstrategien. Fernstudieneinheit 23. Berlin, München, Wien, Zürich, New York: Goethe Institut.

Chromcak, Pavel (2008): Sprachlernberatung. Stand des Fachdiskurses. Saarbrücken: VDM Verlag.

Claußen, Tina (2009): Strategietraining und Lernberatung. Tübingen: Stauffenberg Verlag.

Ferrance, Eileen (2000): Action Research. Providence: LAB Northeast and Islands Regional Educational Laboratory at Brown University.

Funk, Hermann u.a. (2014): Aufgaben, Übungen, Interaktion. DLL. München: Langenscheidt.

Grieshammer, Ella u.a. (2016): Zukunftsmodell Schreibberatung. Eine Einleitung zur Begleitung von Schreibenden im Studium. 4-3. korr. Auflage. Hohengehren: Schneider Verlag.

Gürgür, Hasan (2016): Eylem Araştırması. In: Saban, Ahmet / Ersoy, Ali (Hrsg.): Eğitimde Nitel Araştırma Desenleri. Ankara: Anı Yayıncılık, 1-50.

Johnson, Andrew P. (2015): Eylem Araştırması El Kitabı. (übers. v. Uzuner, Yıldız / Özten Anay, Meltem). Ankara: Anı Yayıncılık.

Käpplinger, Bernd / Rohs, Matthias (Hg.) (2014): Lernberatung in der beruflich-betrieblichen Weiterbildung. Konzepte und Praxisbeispiele für die Umsetzung. Leibniz-Zentrum für Lebenslanges Lernen (DIE). www.die-bonn.de/doks/2014-lernberatung-01.pdf (Letzter Zugriff: 25.05.2021) 
Karaoğlu-Bircan, Çağlayan (2020): Sprachlernberatung in der Deutschlehrerausbildung. Schreiben im Fokus. Dissertationsarbeit an dem Institut für Sozialwissenschaften Universität Trakya. Aufrufbar unter: https://tez.yok.gov.tr/UlusalTezMerkezi/tezSorguSonucYeni.jsp (Letzter Zugriff: 27.04.2021)

Kelle, Udo / Erzberger, Christian (2000): Qualitative und quantitative Methoden: kein Gegensatz. Oppenheim: Na-Verlag.

Kleppin, Karin (2002): Fehler und Fehlerkorrektur. Fernstudieneinheit 19. Berlin u.a.: Langenscheidt, München Goethe Institut.

Kleppin, Karin (2010): Lernberatung. In: Krumm, Hans-Jürgen u.a. (Hg.): Deutsch als Fremd- und Zweitsprache, 2. Halbband. Ein internationales Handbuch. Tübingen und Basel: Francke, 11621166

Köksal, Handan (2019): Magisterarbeitsbetreuung im Umfeld der Sprachlernberatung. Retrospektive Ergebnisse aus der Universität Trakya. In: Muttersprache 129, 75-92.

Lubzyk, Jessica u.a. (2016): Die Schreiblernberatung als integraler Bestandteil des Nürtinger Beratungsmodells IBIS. In: Knorr, Dagmar (Hg.): Universitätskolleg-Schriften Band 13. Akademisches Schreiben - Vom Qualitätspakt Lehre 1 geförderte Schreibprojekte. Hamburg: Universität Hamburg, 157-162. https:/www.universitaetskolleg.uni-hamburg.de/publikationen/ukschriften-013.pdf (Letzter Zugriff: 28.05.2021).

Pätzold, Henning (2006): Pädagogische Beratung und Lernberatung. In: Qualitätssicherung an Schulen. Hg. v. Rolf, Arnold / Gómez-Tutor, Claudia, s.135-175. Verlag: Auer https://www.researchgate.net/publication/281744989_Padagogische_Beratung_und_LernB (Letzter Zugriff: 29.04.2021)

Rogers, Carl-Ransom (1992): The processes of therapy. In: Journal of Consulting and Clinical Psychology 60(2), 163-164. https://doi.org/10.1037/0022-006X.60.2.163 (Letzter Zugriff: 28.03.2021).

Saunders, Constanze (2015): Online-Sprachlernberatung im universitären Kontext: Szenarien auf dem Prüfstand. Eine fallbasierte Longitudinalstudie im Rahmen von Aktionsforschung. (Unveröffentlichte Dissertation). Universität Leipzig. https:/ul.qucosa.de/api/qucosa\%3A13260/attachment/ATT-0/ (Letzter Zugriff: 28.04.2021).

Schiffauer, Werner u.a. (2009): Einführung in die Methoden der empirischen Sozialforschung. https:/www.kuwi.europa-uni.de/de/lehrstuhl/vs/polsoz/Lehre-Archiv/lehre-ws09/methodenempirie/Vorlesung_15_10_2009.pdf (letzter Zugriff: 30.06.2020).

Siebert, Horst (2006): Selbstgesteuertes Lernen und Lernberatung. Konstruktivistische Perspektiven. 2.überarbeitete Auflage. Augsburg: Ziel Verlag.

Tok, Emine (2015): Sprachlernberatung für DaF-Studierende. In: Toprak, Metin/ Karabağ, İmran (Hrsg.): Migration und kulturelle Diversität - Tagungsbeiträge des XII. Internationalen Türkischen Germanistik Kongresses. Bd. II: Sprachwissenschaft und Sprachdidaktik. Frankfurt am Main: Peter Lang, 279-287.

Tressel, Jana (2017): Anknüpfungen an die Grundschule, das heißt Bildergeschichten-eine Interviewstudie zur Sicht von Gymnasial-und Grundschullehrkräften auf den Schreibunterricht der Grundschule. (Unveröffentlichte Dissertation). Leuphana Universität, Lüneburg. https://pubdata.leuphana.de/frontdoor/deliver/index/docId/831/file/Dissertation_Tressel.pdf (Letzter Zugriff 27.04.2021).

Vater, Heinz (2001): Einführung in die Textlinguistik. Struktur und Verstehen der Texte. München: Wilhelm Fink Verlag.

Vogler, Stefanie / Hoffmann, Sabine (Hg.) (2011): Sprachlernberatung für DaF. Berlin: Frank \& Timme. 